Pre-Amplifier Module for Laser Inertial Confinement Fusion

J. E. Heebner, M. W. Bowers

March 4, 2008 
This document was prepared as an account of work sponsored by an agency of the United States government. Neither the United States government nor Lawrence Livermore National Security, LLC, nor any of their employees makes any warranty, expressed or implied, or assumes any legal liability or responsibility for the accuracy, completeness, or usefulness of any information, apparatus, product, or process disclosed, or represents that its use would not infringe privately owned rights. Reference herein to any specific commercial product, process, or service by trade name, trademark, manufacturer, or otherwise does not necessarily constitute or imply its endorsement, recommendation, or favoring by the United States government or Lawrence Livermore National Security, LLC. The views and opinions of authors expressed herein do not necessarily state or reflect those of the United States government or Lawrence Livermore National Security, LLC, and shall not be used for advertising or product endorsement purposes.

This work performed under the auspices of the U.S. Department of Energy by Lawrence Livermore National Laboratory under Contract DE-AC52-07NA27344. 


\section{Pre-Amplifier Module for Laser Inertial Confinement Fusion Stable, uniform, precise, high-gain interchangeable module} UCRL-MI-?????

\section{Lawrence Livermore National Laboratory}
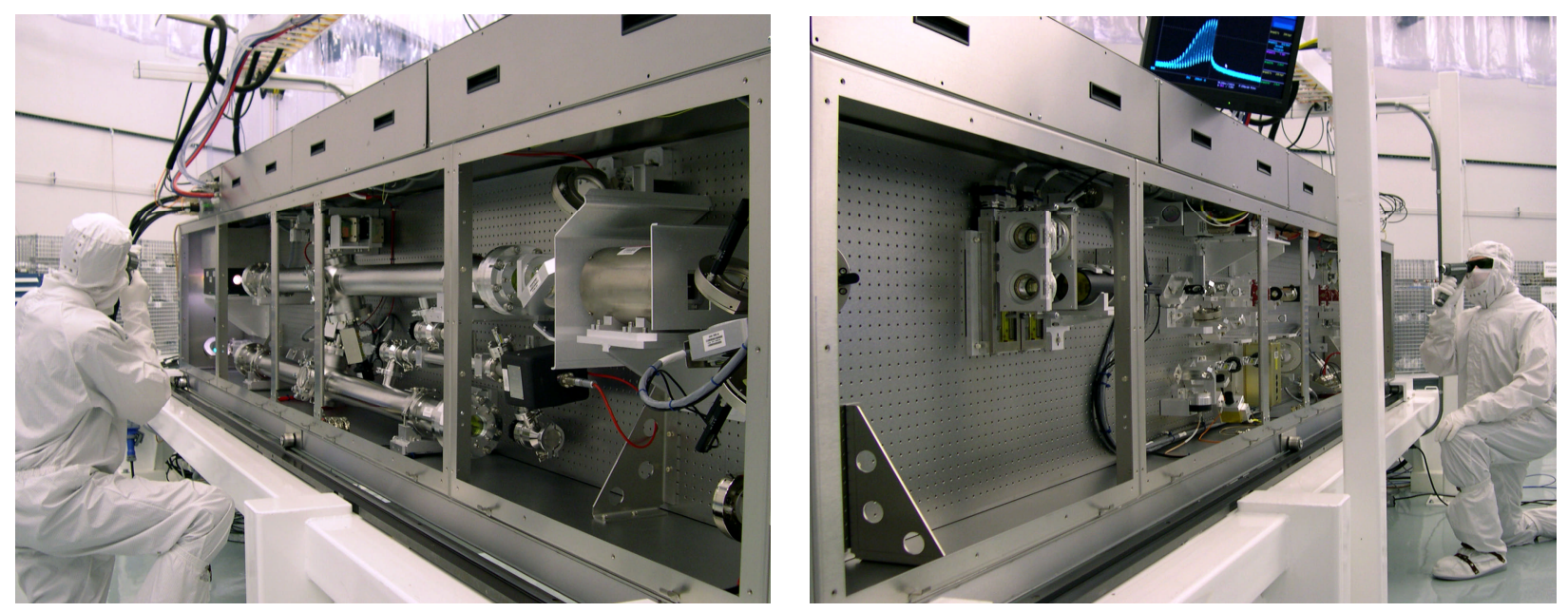

The Pre-Amplifier Modules (PAMs) are the heart of the National Ignition Facility (NIF), providing most of the energy gain for the most energetic laser in the world. Upon completion, NIF will be the only laboratory in which scientists can examine the fusion processes that occur inside stars, supernovae, and exploding nuclear weapons and that may someday serve as a virtually inexhaustible energy source for electricity. Consider that in a fusion power plant 50 cups of water could provide the energy comparable to 2 tons of coal.

Of paramount importance for achieving laser-driven fusion ignition with the least energy input is the synchronous and

The National Ignition Facility requires 48 interchangeable $P A M s$.

Each PAM provides 100 decibels (dBs) of optical gain- equivalent in audio terms to the difference between a whisper and a jet engine on takeoff.

Energy output of each PAM is up to 10 Joules.

Shot-to-shot energy stability is $<1 \%$ symmetric compression of the target fuel-a condition known as laser power balance. NIF's 48 PAMs thus must provide energy gain in an exquisitely stable and consistent manner. While building one module that meets performance requirements is challenging enough, our design has already enabled the construction and fielding of 48 PAMs that are stable, uniform, and interchangeable.

PAM systems are being tested at the University of Rochester's Laboratory for Laser Energetics, and the Atomic Weapons Enterprise of Great Britain has purchased the PAM power system. 



$\begin{array}{ll}\text { 1. Submitting Organization: } & \text { Lawrence Livermore National Laboratory } \\ \text { Address: } & 7000 \text { East Avenue, Mail Code L-464 } \\ \text { City: } & \text { Livermore } \\ \text { State: } & \text { California } \\ \text { Zip code: } & 94550 \\ \text { Country: } & \text { USA } \\ \text { Submitter's name: } & \text { John E. Heebner } \\ \text { Phone: } & 925-422-5474 \\ \text { Fax: } & 925-422-7748 \\ \text { Email: } & \text { heebner1@1lnl.gov }\end{array}$

AFFIRMATION: I affirm that all information submitted as a part of, or supplemental to, this entry is a fair and accurate representation of this product.

Submitter's signature:

\section{Joint entry with:}

N.A.

\section{Product name:}

Pre-Amplifier Module (PAM) for Laser Inertial Confinement Fusion

\section{Brief description:}

Ultra-stable, uniform, and interchangeable multi-Joule, 100-dB laser amplifiers provide most of the optical gain for the most energetic laser in the world to be used to test inertial confinement fusion.

\section{When was this product first marketed or available for order?}

The Pre-Amplifier Module (PAM) technology was first available for order in April, 2007. 


\section{Inventor or Principal Developer}

$\begin{array}{ll}\text { Lawrence Livermore National Laboratory } \\ \text { Developer Name: } & \text { John E. Heebner } \\ \text { Position: } & \text { Lead Scientist } \\ \text { Organization: } & \text { Lawrence Livermore National Laboratory } \\ \text { Address: } & 7000 \text { East Avenue, Mail Code L-464 } \\ \text { City: } & \text { Livermore } \\ \text { State: } & \text { California } \\ \text { Zip Code: } & 94550 \\ \text { Country: } & \text { USA } \\ \text { Phone: } & 925-422-5474 \\ \text { Fax: } & 925-422-7748 \\ \text { Email: } & \text { heebner1@1lnl.gov }\end{array}$

Additional contributors are listed in Appendix A.

\section{Product price}

There are no current plans to bring the PAM to market; however, its overall value was demonstrated by the decision of the Laboratory for Laser Energetics (LLE) at the University of Rochester and the Atomic Weapons Establishment (AWE) of the United Kingdom, to purchase NIF Preamplifier hardware rather than design and build their own systems or procure commercial products. LLE purchased a complete PAM/Power Supply system and AWE bought several NIF Preamplifier Power Supplies to support their experimental programs.

\section{Do you hold any patents or patents pending on this product?}

Raymond Beach et al. "Hollow Lensing Duct" U.S. Patent 6,160,934 


\section{Describe your product's primary function as clearly as possible. What does it do? How does it do it? What theories, if any, are involved?}

Lawrence Livermore National Laboratory's Pre-Amplifier Modules (PAMs) are modular and interchangeable subsystems that provide most of the energy for the National Ignition Facility (NIF). Each of the 48 PAM amplifies a seed pulse from less than a nanojoule to as much as 10 joules, or a factor of 10 billion. The pulse is then split four ways to feed four Main Amplifiers, where the energy of each is increased again, this time by a factor of a few thousand. All 192 beamlines combine at the target chamber for a total energy that exceeds a megajoule. This enormous energy is essential for achieving fusion ignition.

\section{Significance of the National Ignition Facility}

Since the late 1940 's, researchers have used magnetic fields to confine hot, turbulent mixtures of ions and free electrons called plasmas so they can be heated to temperatures of 100 to 300 million Kelvins (180 million to 540 million degrees Fahrenheit). Under those conditions, positively charged deuterium nuclei (containing one neutron and one proton) and tritium nuclei (two neutrons and one proton) can overcome the repulsive electrostatic force that keeps them apart and "fuse" into a new, heavier helium nucleus with two neutrons and two protons. The helium nucleus has a slightly smaller mass than the sum of the masses of the two hydrogen nuclei, and the difference in mass is released as kinetic energy, according to Albert Einstein's famous formula $\mathrm{E}=\mathrm{mc}^{2}$. The energy is converted to heat as the helium nucleus, also called an alpha particle, and the extra neutrons interact with the material around them.

In the 1970's, scientists began experimenting with powerful laser beams to compress and heat the hydrogen isotopes in capsules to the point of fusion. The fusion reaction propagates outward through the cooler, outer regions of the capsule much more rapidly than the capsule can expand. Magnetic fields are unnecessary as the plasma is confined by the inertia of its own mass. The technique is called inertial confinement fusion, or ICF.

The National Ignition Facility (NIF) is designed to produce fusion burn and energy gain using a novel indirect approach to ICF on a heretofore-unprecedented scale. NIF's intense laser beams, focused into a tiny gold cylinder called a hohlraum, will generate a "bath" of soft X-rays that will compress a tiny hollow shell filled with deuterium and tritium to 100 times the density of lead. As a 
result, a temperature of more than 100 million degrees Celsius and pressures 100 billion times the Earth's atmosphere will be achieved. Under these extreme conditions, the fuel core will undergo a self-sustaining thermonuclear burn or "ignition" releasing 10 to 100 times more energy than the amount deposited by the laser beams.

Experiments on NIF beginning in 2010 will be the first using ICF to generate more energy released from the fusion fuel than the laser energy used to produce the fusion reaction. Creating inertial confinement fusion and energy gain in the NIF target chamber will be a significant step toward making fusion energy viable in commercial power plants. Determining the minimum energy needed to start the fusion process is critical to determining the viability of inertial fusion energy. Thus NIF can provide the basis for evaluating future decisions about inertial fusion energy development facilities and programs.

In a fusion power plant, the heat from the fusion reaction would be used to drive a steam-turbine generator to produce electricity. The simplest fusion fuels, the heavy isotopes of hydrogen (deuterium and tritium), are derived from water and the metal lithium, a relatively abundant resource. The fuels are virtually inexhaustible - one in every 6,500 atoms on Earth is a deuterium atom - and they are available worldwide. One gallon of seawater would provide the equivalent energy of 300 gallons of gasoline; fuel from 50 cups of water contains the energy equivalent of 2 tons of coal. A fusion power plant would produce no pollutants or climate-changing gases, as well as considerably lower amounts and less environmentally harmful radioactive byproducts than current nuclear power plants. NIF will not be used to generate electricity. However, by demonstrating fusion ignition and energy gain in the laboratory, NIF experiments should bring fusion energy a major step closer to being a viable source of virtually limitless energy. 


\section{Beamlines}

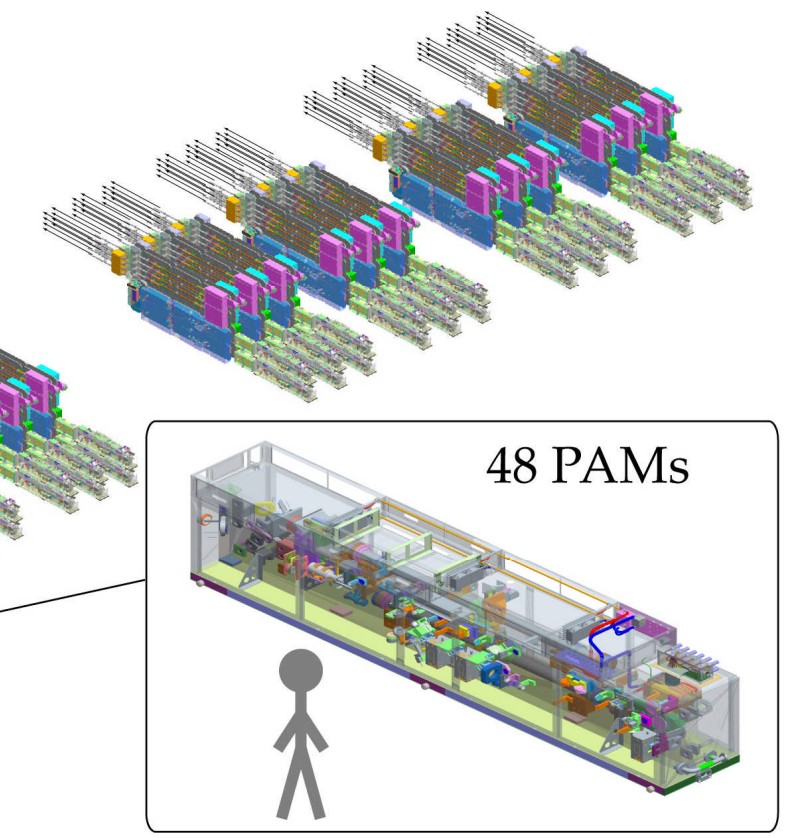

Figure 1. CAD rendering of the system of 48 PAMs and four-way branching optics feeding 192 main beamlines (arranged in 24 bundles of 8 in upper left). The insert in the lower right shows a blowup of a PAM with a human figure included to convey scale.

\section{NIF Amplification}

NIF will rely on 192 pulsed laser beams (Figure 1) to compress targets, resulting in pressures and densities required to initiate thermonuclear burn. The laser amplifiers provide optical gain of over 13 orders of magnitude from nanojoules to 10 s of kilojoules per beamline. Inside NIF's stadiumsize building, laser components shape and smooth a seed pulse, amplify it more than a quadrillion times, and direct it at a tiny target precisely centered in the target chamber. This process is replicated simultaneously 192 times, with all beamlines converging on the target chamber. The combined action of gain ( $>13$ orders) and multiplexing of beamlines ( $>2$ orders) results in an energy rampup across 15 orders of magnitude. Figure 2 illustrates the critical role that the 48 PAMs play in delivering most of this amplification with its two internal stages: the Regenerative amplifier (Regen) and Multi-Pass Amplifier (MPA). 


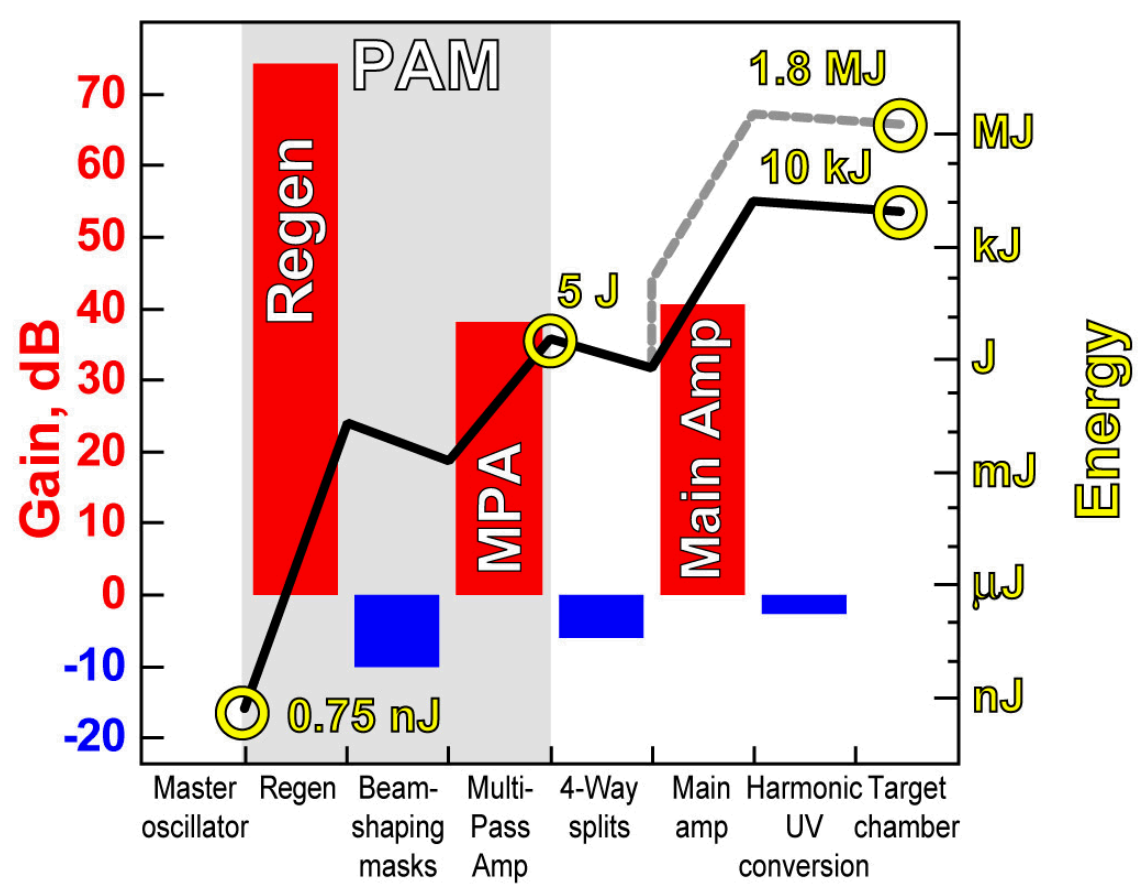

Figure 2. An overview of the NIF gains and energies by subsystem. The red bars represent laser amplifier gain and the blue bars represent losses due to beam-shaping, beamline spliting and harmonic conversion elements. The solid black line denotes the accumulated energy per beamline. The dashed line represents the combined energy of 192 beamlines. Fifteen orders of magnitude are traversed in the rampup of energy delivered to the fusion target. More than ten of these orders of magnitude are delivered by the PAMs.

\section{The PAMS at Work}

Each PAM is a precision, two-stage high-gain laser amplifier with a gain of over 10 billion. PAMs have been designed to enable the construction of the largest and most energetic laser ever built. Of paramount importance for achieving ignition on NIF with the lowest possible input energy is the synchronous and symmetric compression of the target fuel, a condition known as laser power balance. This in turn demands that the 48 PAMs provide their gains in an exquisitely stable and consistent manner. All PAMs have met a demanding set of requirements including energy and pointing stability, beamand pulse shape uniformity / conformity, and interchangeability.

Production, installation, and qualification of the 48 PAMs required for NIF concluded in November 2007. Extra PAMs are being produced both for ready-to-swap spares on NIF and for external customers. 
The two stages of the PAM are built up on opposite sides of a vertically-oriented optical breadboard as shown in the CAD rendering of figure 3. The first stage Regenerative Amplifiers (Regens) in each of the 48 PAMs are designed to operate at $1 \mathrm{~Hz}$ in a highly stable regime for energy, spatial pulse shape, temporal shape, and optical pre-pulse noise. The design of the Regen is such that fluctuations in the injected pulse energy as high as $20 \%$ deviating from a nominal setpoint of 0.75 nanojoules from the master oscillator are reduced to less than $0.25 \%$ at the 25 millijoules Regen output. Following the Regen, the Gaussian beam is reformatted into a square beam with a pre-compensated shape tailored for the Main Amplifiers. The beam then transits through the second stage Multi-Pass Amplifier (MPA) system in each PAM where it is amplified by a factor of 10,000 up to 10 Joules. The MPA is designed to minimize the possibility of parasitics (unwanted selfoscillations due to inadverdent feedback above the $0.01 \%$ level) and pencil beams (undesired amplification of reflections from transmitting surfaces). The PAM outputs have highly stable beam pointing characteristics and excellent beam quality.

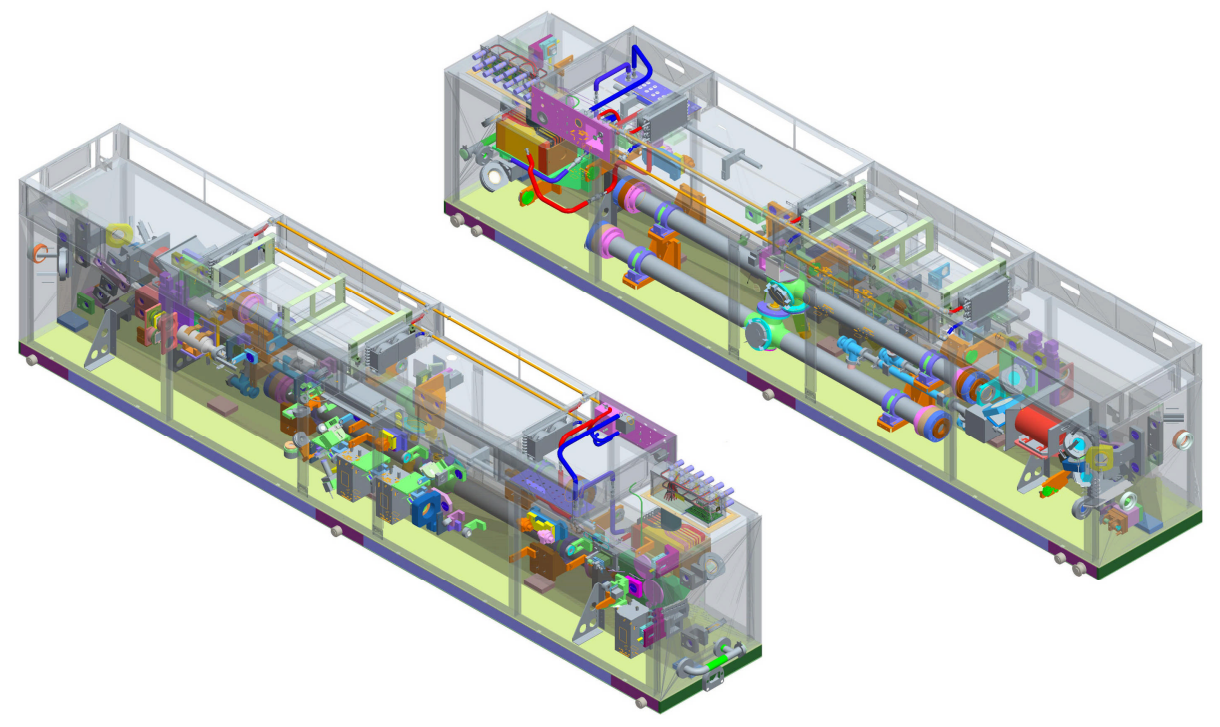

Figure 3. CAD rendering of both sides of the PAM. A view from the Regenerative Amplifier side is illustrated on the left and a view from the Multi-Pass Amplifier is on the right. The PAM dimensions are $15-\mathrm{ft} \times 2-\mathrm{ft} \times 3-\mathrm{ft}$.

\section{The Regen}

The NIF PAM Regen consists of a laser diode pumped neodymium-doped phosphate glass rod (LG770), and a folded 45-ft long (45-ns round trip) resonant cavity. The 5-mm-diameter amplifier rod is pumped at $800 \mathrm{~nm}$ by a $4-\mathrm{kW}$ diode array for 0.4 milliseconds. Pump light is concentrated into the rod via use of a cored lens and hollow duct. The arrangement allows for a diffuse, end- 
pumped configuration while maintaining passage of the $1053 \mathrm{~nm}$ cavity beam. The pumped end of the rod is antireflection-coated at both wavelengths while the other end is coated for low reflection at $1053 \mathrm{~nm}$ and high reflection at $800 \mathrm{~nm}$. This amplifier head geometry enables positioning of the rod at a dynamically stable location, where the mode size is insensitive to surface figure perturbations on the rod facets and thermal lensing in its bulk. The cavity is folded with mirrors to condense the $45 \mathrm{ft}$ long cavity. Figure 4 displays a schematic of the unfolded resonant cavity along with photographs of the amplifier head assembly and rod.
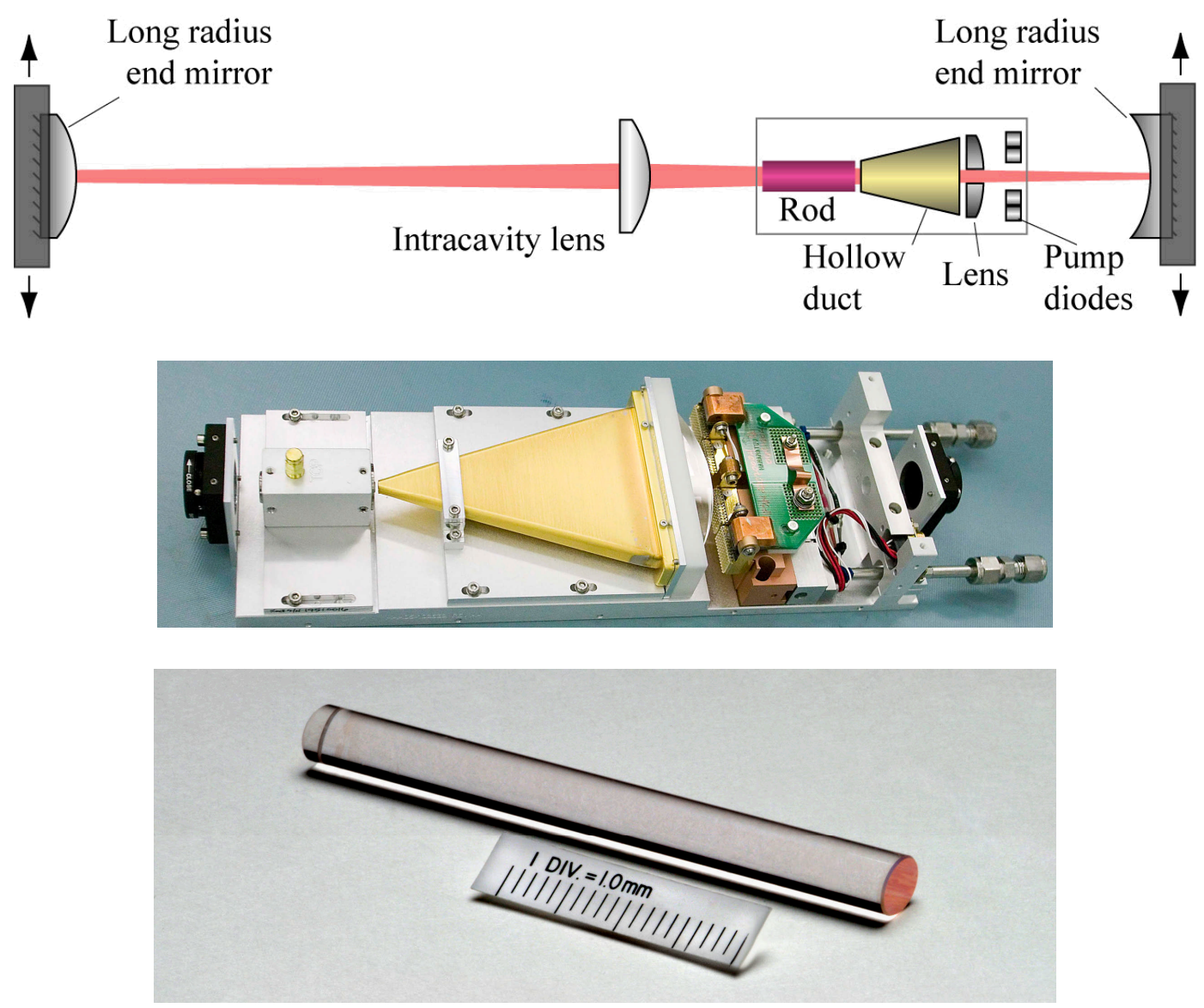

Figure 4. a) Unfolded regenerative amplifier resonant cavity. A hollow lens duct end pumped geometry is used to concentrate $800-\mathrm{nm}$ pump light from a 4-kW diode array into the neodymium-doped phosphate glass rod. Translation-mounted curved end mirrors with a long radius of curvature are employed for precise alignment. b) Photograph of the hollow lens duct amplifier head assembly. c) Photograph of the 5-mmdiameter, 50-mm-long neodymium-doped phosphate glass (LG770) rod. 
The Regen resonant cavity was designed with the goals of stability, uniformity, and reliability with minimized hardware complexity. Unfolded, the cavity consists of two curved end mirrors, a rod and an intra-cavity lens. Numerous cavity configurations were considered and evaluated for:

- Beam size stability \& consistency (impacted by surface figure errors)

- Steering alignment insensitivity (in the presence of vibrations and drift)

- Efficient energy extraction (optimally filling the rod with the beam size)

- Robustness against optical damage (ensuring that no focused beam "hotspots" exist)

Due to an imposed constraint of an unusually long cavity to accommodate up to 33 ns pulses, meeting these goals was challenging since even weak focal lensing due to pump-induced thermal gradients and surface figure errors can contribute to large changes in beam size for unoptimized cavity configurations. For many cavity designs, small changes in rod focal power change the beam area by several percent, in turn changing the output energy proportionally. This sensitivity impacts both the stability of any one PAM and the uniformity across PAMs:

- Shot to shot pumping variations lead to beam size instability that in turn leads to energy instability.

- Surface figure inconsistencies across manufactured rods can lead to disparities in the mode shape from PAM to PAM.

As a function of rod focal power, there are always two zones supporting geometrically stable light paths. Within these geometrically stable zones, there exists an optimal plateau where the change in beam size in the rod due to changes in the rod focal power is zero. One of these zones is less sensitive to beam decentering perturbations while the other is less sensitive to beam steering perturbations. Because the optics employed for folding the cavity are mirrors that unavoidably convert mechanical vibrations and drifts into tilts, we chose an optimal configuration in the steering-insensitive zone. The curvatures of the two end mirrors and focal power of the intra-cavity lens then became degrees of freedom that allowed us to remain optimized in this zone while maximizing the energy extraction efficiency and simultaneously minimizing hotspots due to an overly focused beam.

Proceeding from an optimized resonant cavity design, numerous iterations were made towards improving the overall stability of the opto-mechanical mounts. The seed pulse from the master oscillator is injected via an optical fiber that is positioned in a novel actuator-free mount consisting of sliding plates. The fold mirrors are likewise adjusted without actuators but rather by nudgerotating the base along one axis and rotating wedged mirrors for steering corrections in the other axis. Finally, the cavity is aligned in an unconventional manner-not with tip/tilt mounts but rather 
by translating the weakly-curved end mirrors. These improvements have resulted in highly precise initial alignment, elimination of actuator drift \& backlash, and overall "set \& forget" operation.

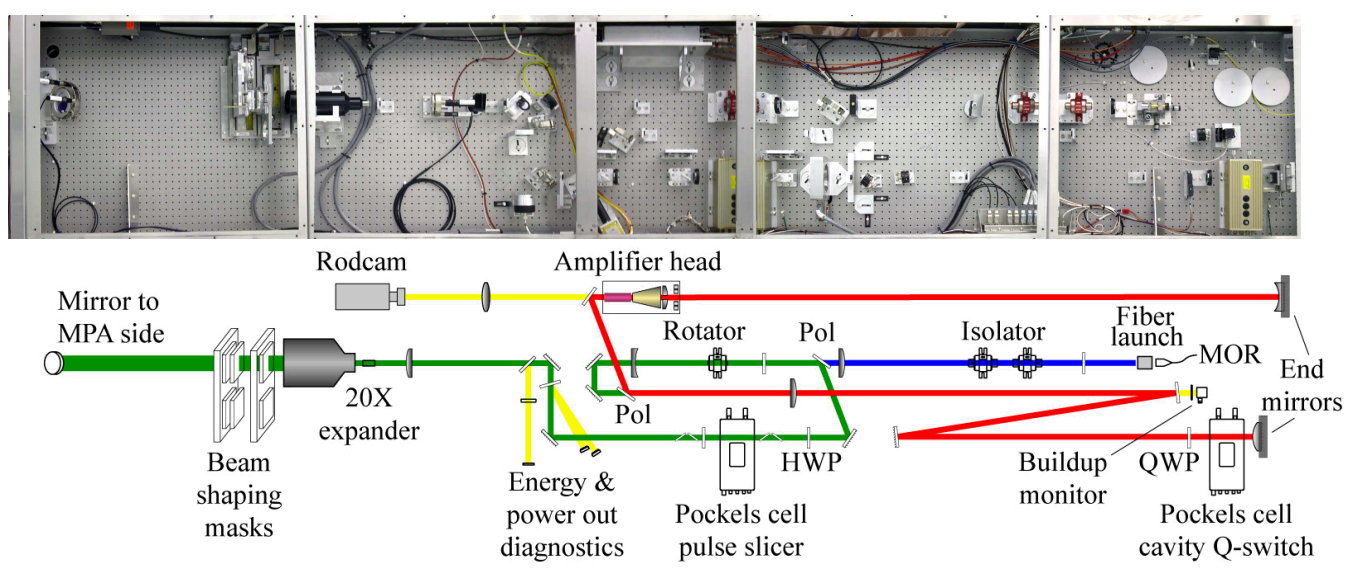

Figure 5. PAM first stage Regenerative Amplifier and Beam Shaping Module layout. The Regenerative amplifier cavity is seeded from the Master Oscillator Room (MOR), Q-switched / cavity-dumped in the resonant cavity, and extracted through a Faraday rotator. The output passes through a pulse slicer and is spatially formatted for injection into the Multi-Pass Amplifier (MPA).

Figure 5 displays a photograph of the Regen resonant cavity along with a schematic identifying key components. A Pockels cell in the cavity is used to Q-switch a tailored seed pulse into and out of the 45-ns cavity. Amplification takes place over 116 passes through the rod with a single-pass net gain factor of $\sim 1.21$. Relatively low gain over many passes results in exquisite energy stability when adjusted for switchout at the peak of the energy buildup $(\sim 25 \mathrm{~mJ})$ where the gain is exhausted (saturated) and is equal to losses. The total path length in the Regen when taking into account the folds and multiple passes is over half a mile and represents two-thirds of the total path delay on the NIF system.

\section{Into the Multi-Pass Amplifier}

The output pulse passes through a Pockels cell slicer (electro-optical time gate) to clean up residual prepulse power leakage up to an extinction level greater than one million to one. The output Gaussian beam is then expanded up to $60 \mathrm{~mm}$ and shaped into a square profile with a serrated apodizer mask. A square profile was chosen to optimally fill the NIF main laser slabs, which are rectangular and tilted at Brewster's angle presenting a square cross-sectional profile. A shaping 
mask is optionally introduced to compensate for nonuniform spatial gain profiles both in the MPA and in the NIF Main Amplifiers. Alignment fiducials are optionally introduced in order to precisely center the beam output. The shaped beam is then passed through a hole in the vertical breadboard onto the second stage of the PAM.

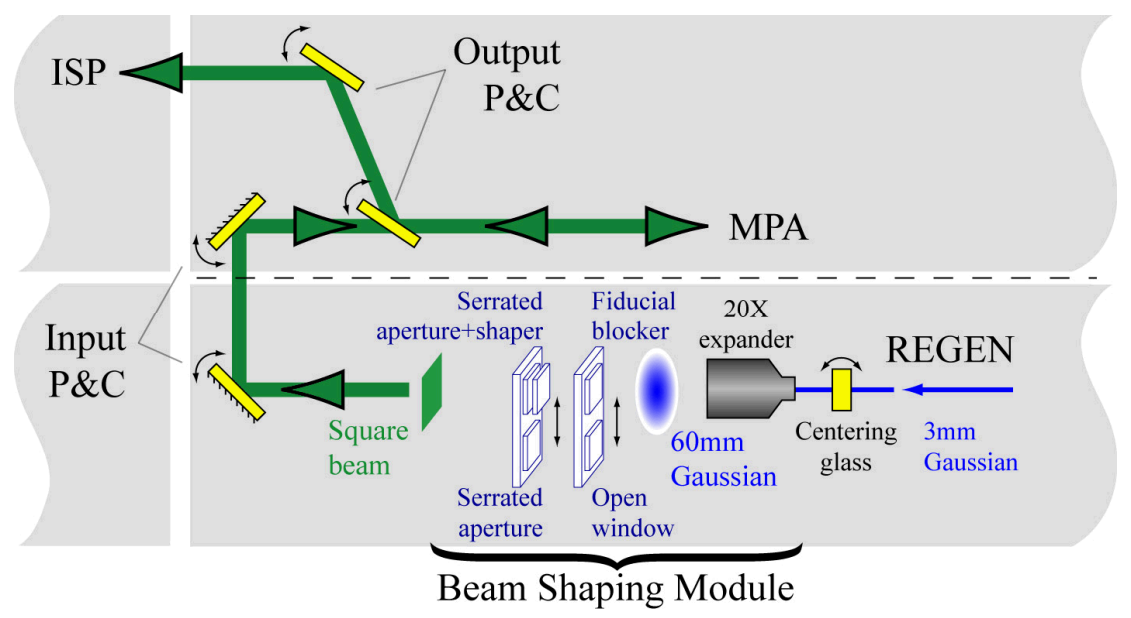

Figure 6. Control points (in yellow) used to align the beam through the Beam Shaping Module, into the MPA and out of the MPA into the ISP. The dashed line represents an unfolding of the breadboards that support the Regenerative Amplifier and MPA. A tiltable glass element is used to center the expanded Regen Gaussian beam onto the shaping masks. Pairs of mirrors and polarizers are used to point $\&$ center $(\mathrm{P} \& \mathrm{C})$ the beam into and out of the MPA.

In order to maintain alignment of the 48 PAMs for the planned 30-year life of NIF, 10 actuated control points were added with automated software routines for adjusting misalignments in centration of the beam on the $20 \mathrm{X}$ expander, pointing and centering into the MPA and pointing and centering out of the MPA. These are illustrated in Figure 6.

The beam is injected through a pair of pointing and centering mirrors and a Faraday rotator before entry into the Multi-Pass Amplifier (MPA). Figure 7 displays a photograph of the MPA along with a schematic identifying key components. The MPA consists of a 4-pass arrangement through a flashlamp-pumped 32-mm diameter by 300-mm-long Neodymium-doped glass (LHG-8) rod as shown in Figure 8. The ends of the rod are tapered to stifle unwanted whispering gallery parasitic modes that rob energy and burn o-ring seals. The flashlamp design incorporates 1.5-inch-thick glass walls for increased mechanical durability and cerium doping to reduce UV emission leading 
to degradation of rubber seals. The voltage supplied to the flashlamps is adjusted near $2.0 \mathrm{kV}$ to target a 4-pass optical gain of $\sim 10,000$.

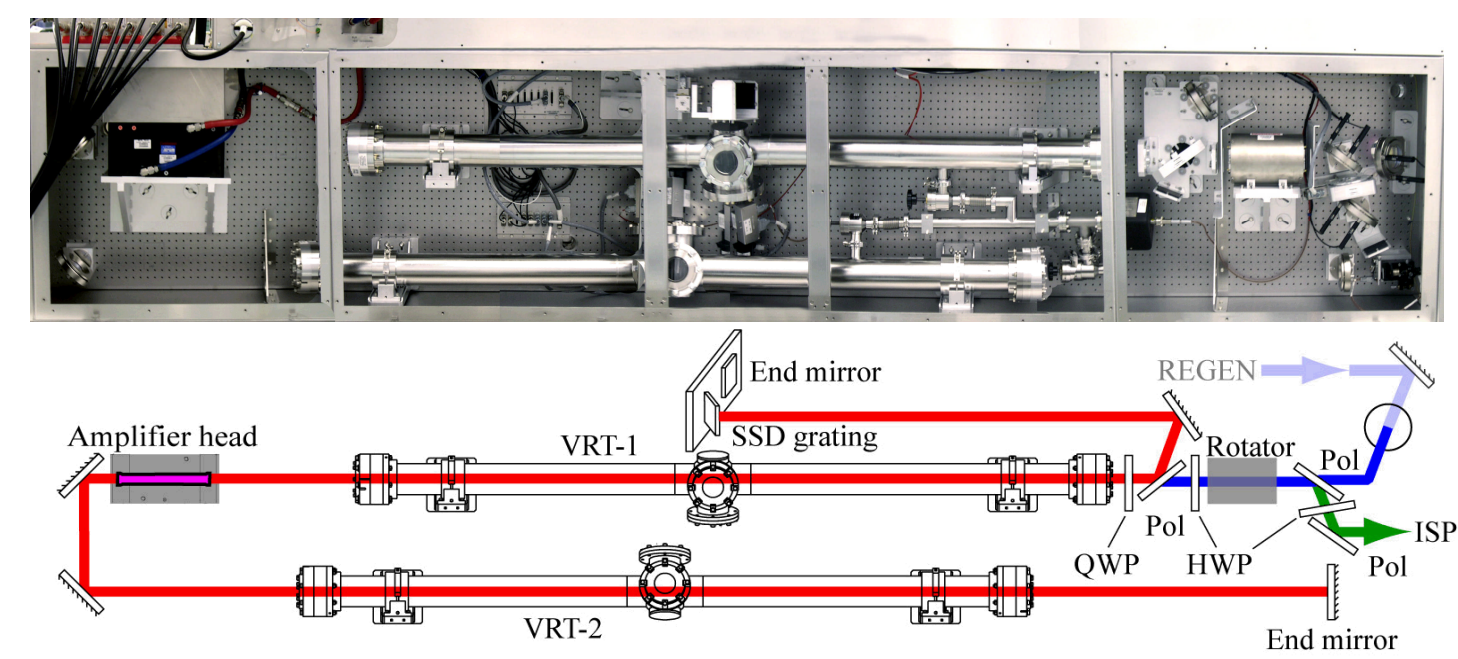

Figure 7. PAM second stage Multi-Pass Amplifier (MPA) geometry. Following the Regenerative Amplifier stage and beam shaping module, the light is injected (blue) through a Faraday rotator into the four-pass amplifier circuit (red). The upper Vacuum Relay Telescope (VRT-1) images the shaped beam onto the flashlamp pumped Nd-glass rod. The lower Vacuum Relay Telescope (VRT-2) images the beam onto a displaced end mirror to avoid spatial hole burning and pulse shape distortion on the second pass. A quarter waveplate (QWP) and polarizer (Pol) enable two more passes through the rod after reflecting off a choice of end mirror or Smoothing by Spectral Dispersion (SSD) grating (oriented at the retro-diffracting Littrow angle) in the upper leg.

The square beam profile is then relay imaged through a pair of two-lens Vacuum Relay Telescopes (VRTs). The VRTs also spatially-filter the serrated edges defined by the beam shaping module yielding a soft-apodized supergaussian edge profile that prevents diffraction induced ripple. The upper telescope (VRT-1) images the shaped beam onto the rod. The lower telescope (VRT-2) images the beam onto a displaced end mirror to avoid spatial hole burning and pulseshape distortion on the second pass. A quarter waveplate enables two more passes through the rod after reflecting off a choice of end mirror or Smoothing by Spectral Dispersion (SSD) grating (oriented at the retro-diffracting Littrow angle) in the upper leg. Phase modulation applied at the maser oscillator working in concert with the angular dispersion of the SSD grating and a Continuous 
Phase Plate (CPP) downstream results in a smoothed farfield spot free of unwanted speckle typically encountered in coherent laser beams.
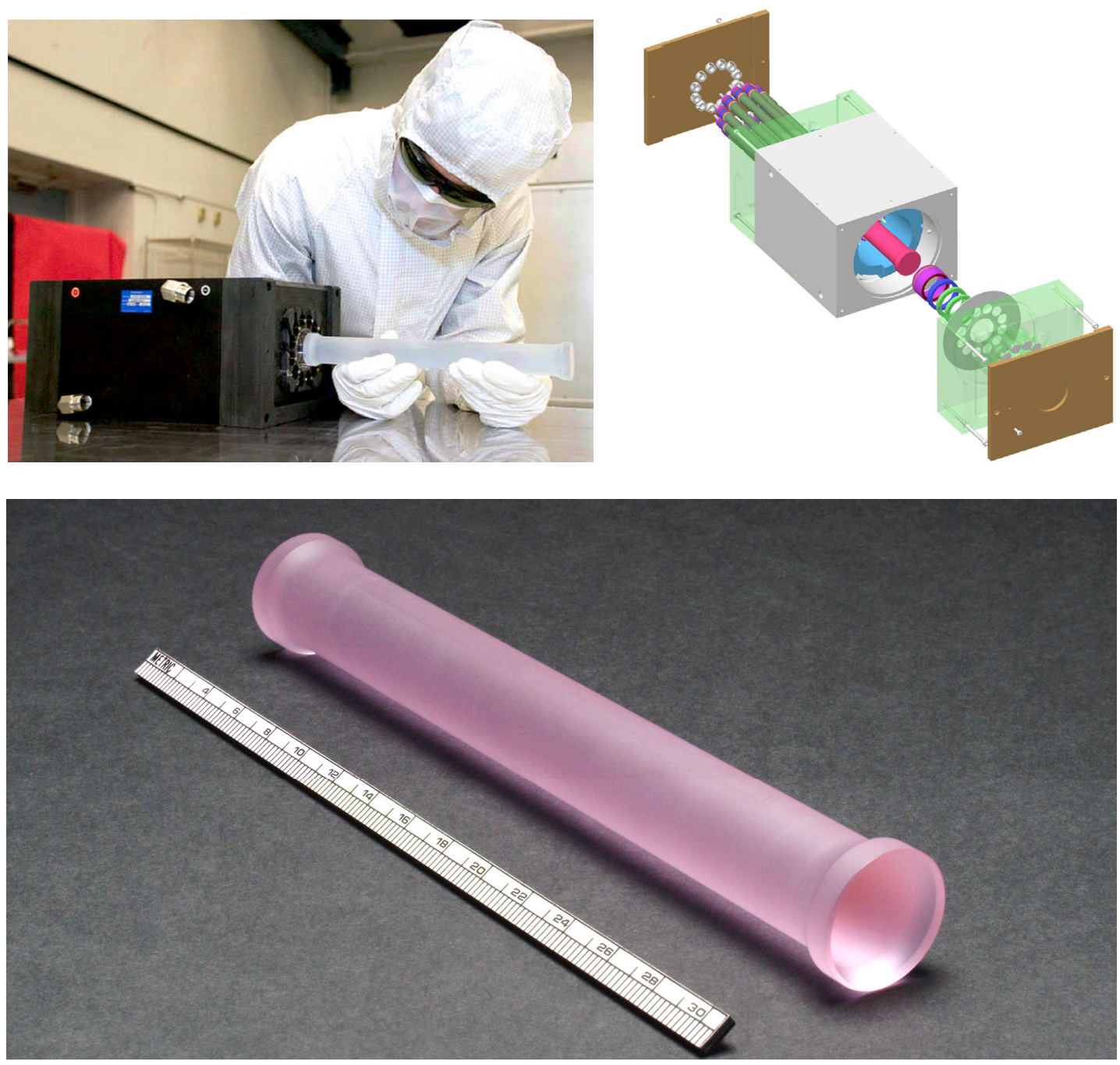

Figure 8. a) Photograph of the MPA amplifier head assembly and b) breakaway diagram displaying the arrangement of the neodymium (Nd) glass rod, 12 flashlamps, pump reflector, light baffles, seals, and endcaps. c) A photograph of the 3-mm-diameter, $300 \mathrm{~mm}$-long neodymium-doped phosphate glass (LHG-8) rod. The rod is manufactured with flared barbell styled ends to minimize whispering gallery parasitic oscillations.

Each VRT contains a 4-element pinhole array such that each of the 4 passes is angularly multiplexed. The lenses are further tilted to suppress unwanted reflections from entering the pinhole array and manifesting themselves as amplified pencil beams. The orientations of the tilts were strategically chosen to minimize net wavefront distortions below 0.5 waves peak-to-valley. 
The system delivers nearly diffraction-limited performance as quantified in Figure 9. Further care has been taken to wedge and/or tilt remaining optical components and baffle mechanical components that give rise to stray reflections that result in parasitic oscillations. The serrated aperture mask is implements a "blue chrome" process which includes both chrome and oxide layers for reduced reflectance. It is further tilted to reduce parasitic mechanisms resulting from reflections off the backside of the mask.

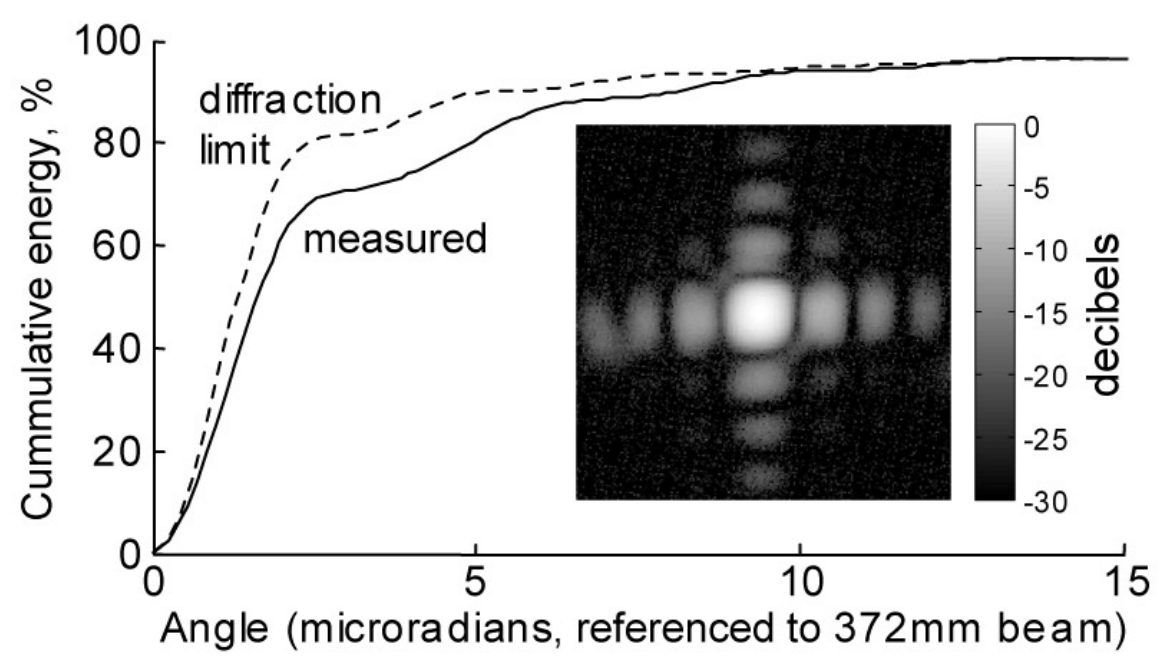

Figure 9. MPA output farfield cumulative angular energy with comparison to the diffraction limit for a perfect square beam. The PAM output beam is $18 \mathrm{~mm}$ square although the angular coordinate is downscaled to correspond to the angular space of the NIF main beam at $372 \mathrm{~mm}$ square. The inset displays the raw farfield spot data displayed on a logarithmic scale. The cross-pattern is nearly the ideal Fourier transform of a square shape.

After four passes and a net gain of 10,000, the beam is amplified with a quadratic gain nonuniformity that is approximately 2.5:1 after 4 passes. The output beam passes through a pair of motorized polarizers that are used to clean up the polarization, attenuate the output in conjunction with a motorized half-waveplate, and make fine corrections to the pointing and centering of the output beam.

The system of 48 PAMs are subject to a stringent set of requirements. A shot-to-shot RMS energy stability of $1 \%$ is achieved when operated near maximum energy output in the saturating regime. The primary sources of instability derive from the Regen $(0.25 \%)$, spatial jitter at the mask 
$(0.25 \%)$, and the MPA gain (0.9\%). The nearfield contrast (spatial noise) is of the order of $3.5 \%$ and deviation from a prescribed shape is $+/-5 \%$. A sample nearfield beam profile is displayed in Figure 10.
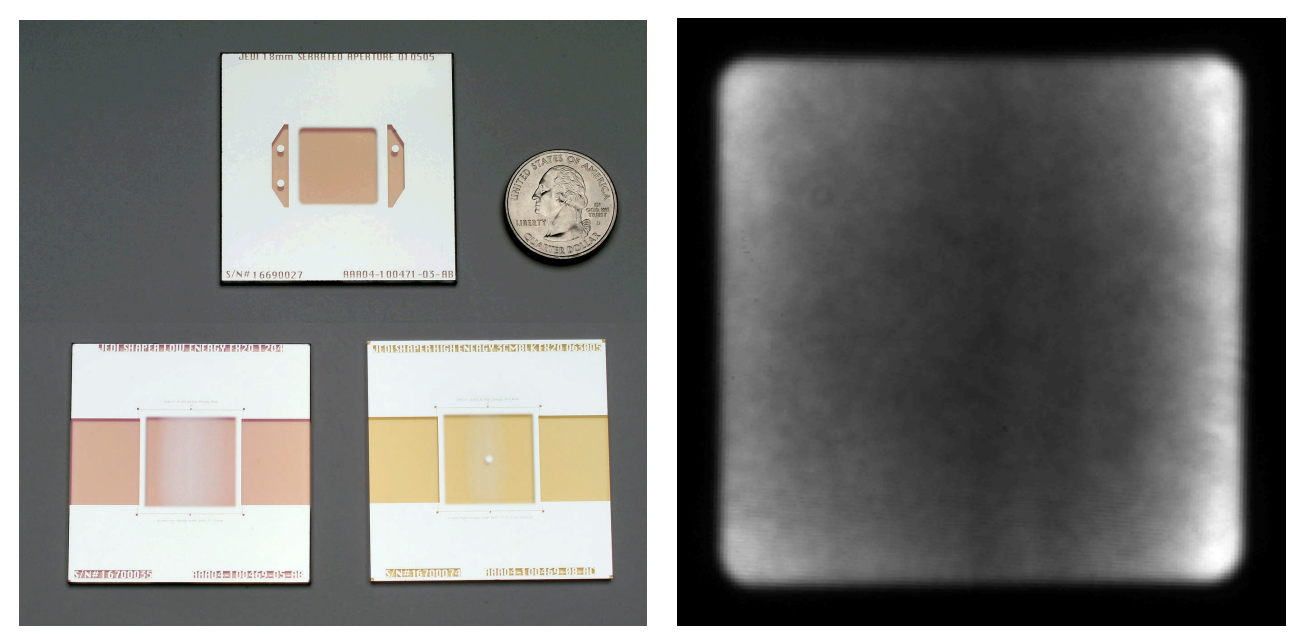

Figure 10. a) Beam shaping masks including the serrated apodizer, anti-gaussian mask and anti-gaussian mask with a fiducial. B) MPA output nearfield image when passed through a serrated apodizer, an anti-gaussian mask and the MPA. High frequency contrast (spatial noise) is typically $\sim 3.5 \%$ on all PAMs. A chrome serrated apodizer mask defines the beam's soft edges. The beam profile is further shaped by a second mask with suppressed fluence near beam center including two dark vertical stripes to precompensate for higher gains in these regions within the main amplifier laser slabs downstream.

All mounts on the MPA were carefully screened and pre-tested for optimum pointing stability. Measurements consistently validate a pointing stability below 10 microradians RMS. Accelerated lifetime tests were performed on motorized components expected to be frequently exercised over the projected 30-year NIF lifetime. These consist of the 10 control points for beam pointing and centering, translation stages that toggle beam shaping and SSD grating options and rotation stages for half-waveplates used to fine-adjust beam energetics. 
10 A. List your product's competitors by manufacturer, brand name and model number.

Livermore's Pre-Amplifier Module (PAM) is a unique system, specifically designed for the National Ignition Facility (NIF), the highest-energy laser in the world. There are similar systems being built at Laser MegaJoule still under construction in France, Atomic Weapons Establishment in Great Britain, and the Laboratory for Laser Energetics in Rochester, NY. The specifications for these systems are unpublished.

There are commercial entities that sell laser systems in nearly the same regime as the PAM. They are listed in the comparative table in the next section. 


\section{B. Comparative Matrix}

\begin{tabular}{|l|c|c|c|c|}
\hline Laser system & $\begin{array}{c}\text { NIF-PAM } \\
\text { Nd:Glass }\end{array}$ & $\begin{array}{c}\text { Continuum } \\
\text { Powerlite } \\
\text { Nd:YAG }\end{array}$ & $\begin{array}{c}\text { Newport } / \\
\text { Spectra- } \\
\text { Physics } \\
\text { Quanta-Ray } \\
\text { Pro } \\
\text { Nd:YAG }\end{array}$ & $\begin{array}{c}\text { Ekspla NL310 } \\
\text { Nd:YAG }\end{array}$ \\
\hline Energy output & 10 Joules & 3 Joules & 2.5 Joules & 1.6 Joules \\
\hline Energy stability & $<1 \%$ RMS & $<1 \%$ RMS & $<2 \%$ RMS & $<1 \%$ RMS \\
\hline Pointing stability & $<10$ microrad & $<30$ microrad & $<25$ microrad & $<100 \mathrm{microrad}$ \\
\hline $\begin{array}{l}\text { Max deviation from prescribed } \\
\text { beam shape }\end{array}$ & $\begin{array}{c}<+/-5 \% \\
\text { Specialized }\end{array}$ & $\begin{array}{c}<+/-40 \% \\
\text { Gaussian }\end{array}$ & $\begin{array}{c}<+/-30 \% \\
\text { Gaussian }\end{array}$ & $\begin{array}{c}<+/-15 \% \\
\text { Gaussian }\end{array}$ \\
\hline Temporal jitter stability & $<0.1 \mathrm{~ns}$ & $<1 \mathrm{~ns}$ & $<0.5 \mathrm{~ns}$ & $<0.5 \mathrm{~ns}$ \\
\hline Pulse width & $0.25-33 \mathrm{~ns}$ & $5-9 \mathrm{~ns}$ & $8-12 \mathrm{~ns}$ & $3-6 \mathrm{~ns}$ \\
\hline Pulse shape & fully adjustable & non-adjustable & non-adjustable & non-adjustable \\
\hline Interchangeability & Yes, proven & No & No & No \\
\hline
\end{tabular}




\section{C. Describe how your product improves upon competitive products or technologies.}

Due to the modular and scalable architecture of NIF, a system of fully interchangeable high gain pre-amplifiers was sought in the early design phases. Requirements for the design and production of the PAMs were put out for competitive bid but no commercial laser company could meet the requirements. While some commercial lasers came close to meeting energy requirements at low energy stabilities and are within factors of 2-5 on allowable spatial and temporal jitter, they did so with simple spatial beam and temporal pulse formats (typically Gaussians, which are the preferred natural modes). The PAMs amplify beam profiles tailored to fit within efficient and scalable square beam architectures and support arbitrarily crafted pulses optimized for target ignition. They do this with a degree of precision unmatched by commercial laser amplifiers.

Progress towards a robust and reproducible design continued even as a PAM factory was being constructed inside the laboratory. In early reviews of the NIF project, the production of 48 "identical" PAMs was considered by many reviewers familiar with the fastidious operation of highenergy laser systems to be an impossible task. The PAMs offer an existence proof that ultra-stable high energy lasers can indeed be produced in a manner that allows them to be used as building blocks in large scale fusion class laser systems. This has further been validated by purchase orders coming from the Laboratory for Laser Energetics (LLE) at the University of Rochester and the Atomic Weapons Establishment (AWE) of the United Kingdom. 


\section{A. Describe the principal applications of this product.}

Several nations (France, Russia, United Kingdom, Germany, Japan, and China to name a few) are pursuing high-energy laser programs. Livermore's National Ignition Facility (NIF) and Photon Science program is the first to reliably mass-produce a Pre-Amplifier Module with the precision energy gain and beam quality control needed for high-energy lasers.

When NIF's full constellation of 192 laser beams are firing, they will compress a tiny target filled with deuterium-tritium fuel to the conditions required for thermonuclear burn, liberating more energy than was required to initiate the fusion reactions. NIF will provide the first laboratory setting for examining fusion reactions that naturally occur only in supernovae, in exploding nuclear weapons, and in our Sun and other stars.

NIF was designed with three specific research goals in mind: to strengthen stockpile stewardship for a safe and reliable nuclear stockpile, show the feasibility of inertial confinement fusion (ICF) as a clean source of energy, and make significant strides in high-energy-density physics to understand the basic physical processes that drive the cosmos. These three missions share the need to prepare materials at extreme conditions - pressures of up to 10 billion megapascals, temperatures of 100 million kelvins, and densities of 100 grams per cubic centimeter. 


\section{B. List all other applications for which your product can now be used.}

As the front end for the world's most energetic laser, the Pre-Amplifier Module offers the precisely controlled laser light essential to expanding the frontiers of high-energy science. NIF will be uniquely capable of producing conditions similar to those in the cores of stars, supernovae, and giant planets. The data from NIF experiments will provide new insights into how the universe operates at both the smallest and the largest scales. 


\section{Summary}

The great promise of a clean, virtually unlimited energy source lies in the production of controlled fusion energy. New, clean sources of energy are of paramount importance to solving many of the country and greater world's problems. However, to date, fusion ignition has only been unleashed in the form of uncontrolled thermonuclear explosions. The National Ignition Facility will enable the first demonstration of controlled inertial confinement fusion where the energy released is greater than the laser energy input. The commercial viability of fusion-based power plants is still poorly understood at this time, but many unanswered questions will soon be finally addressable by NIF. For NIF to operate consistently and reliably, a system of robust Pre-Amplifier Modules is essential. The PAMs set a new defining standard for stability, beam and pulse precision, and consistency for high-energy lasers, enabling interchangeability on a large and upwardly scalable system architecture. 


\section{Organization Data}

13. Contact person to handle all arrangements on exhibits, banquet, and publicity.

$\begin{array}{ll}\text { Name: } & \text { Yvonne King } \\ \text { Position: } & \text { Industrial Partnerships and Commercialization } \\ \text { Organization: } & \text { Lawrence Livermore National Laboratory } \\ \text { Address: } & \text { PO Box 808, L-795 } \\ \text { City: } & \text { Livermore } \\ \text { State: } & \text { California } \\ \text { Zip code: } & 94550 \\ \text { Country: } & \text { USA } \\ \text { Phone: } & 925-422-7299 \\ \text { Fax: } & 925-423-8988 \\ \text { Email: } & \text { king26@1lnl.gov }\end{array}$




\section{List of Appendices}

Appendix A, Additional Contributors

Appendix B, Additional Figures

Appendix C, Multimedia 


\section{Appendix A}

\section{Additional Contributors}

Developer Name: $\quad$ Mark W. Bowers

Position:

Physicist

Organization:

Lawrence Livermore National Laboratory

Phone:

Email:

925-423-0901

bowers5@1lnl.gov

Developer Name:

Position:

Organization: Lawrence Livermore National Laboratory

Phone:

Email: 


\section{Appendix B}

\section{PAM Optics Photographs}

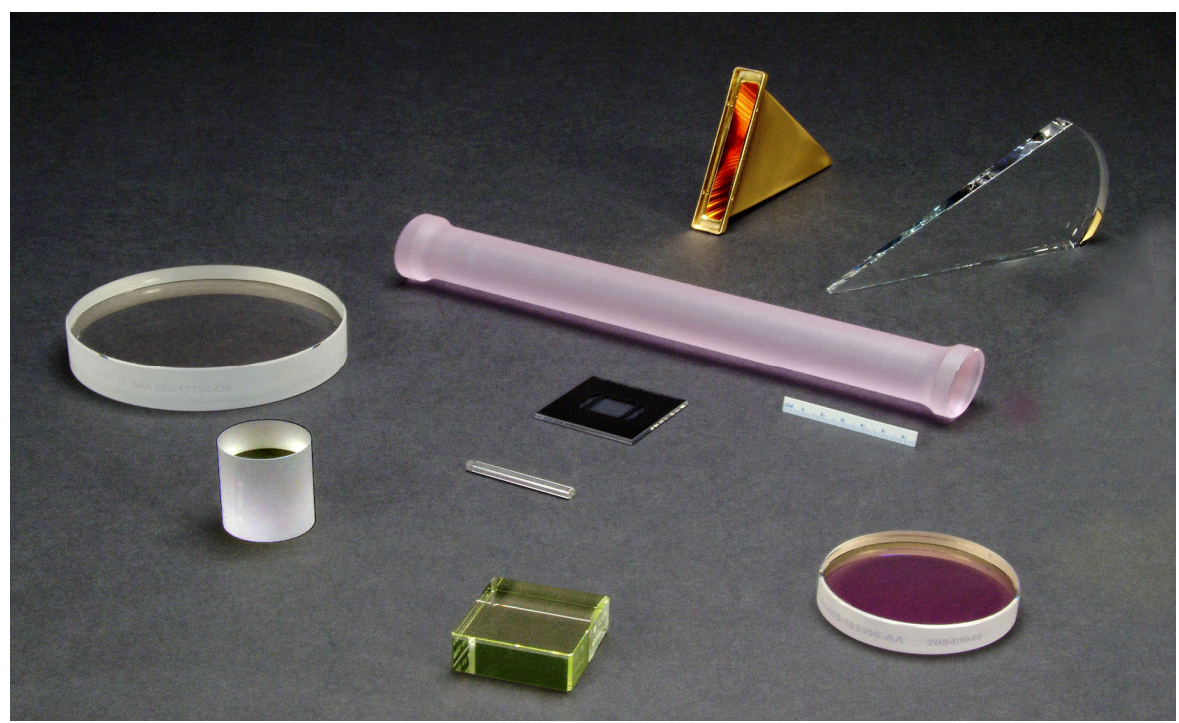

Critical optical components manufactured to tight tolerances specifically for the PAM. From left to right: MPA polarizer, centering glass, MPA barbell rod, regen rod, Faraday rotator FR5 magneto-optic glass element, absorbing blue chrome mask, regen diode pump hollow duct concentrator, regen diode pump glass duct concentrator, MPA end mirror. 


\section{PAM Timing Schematic}

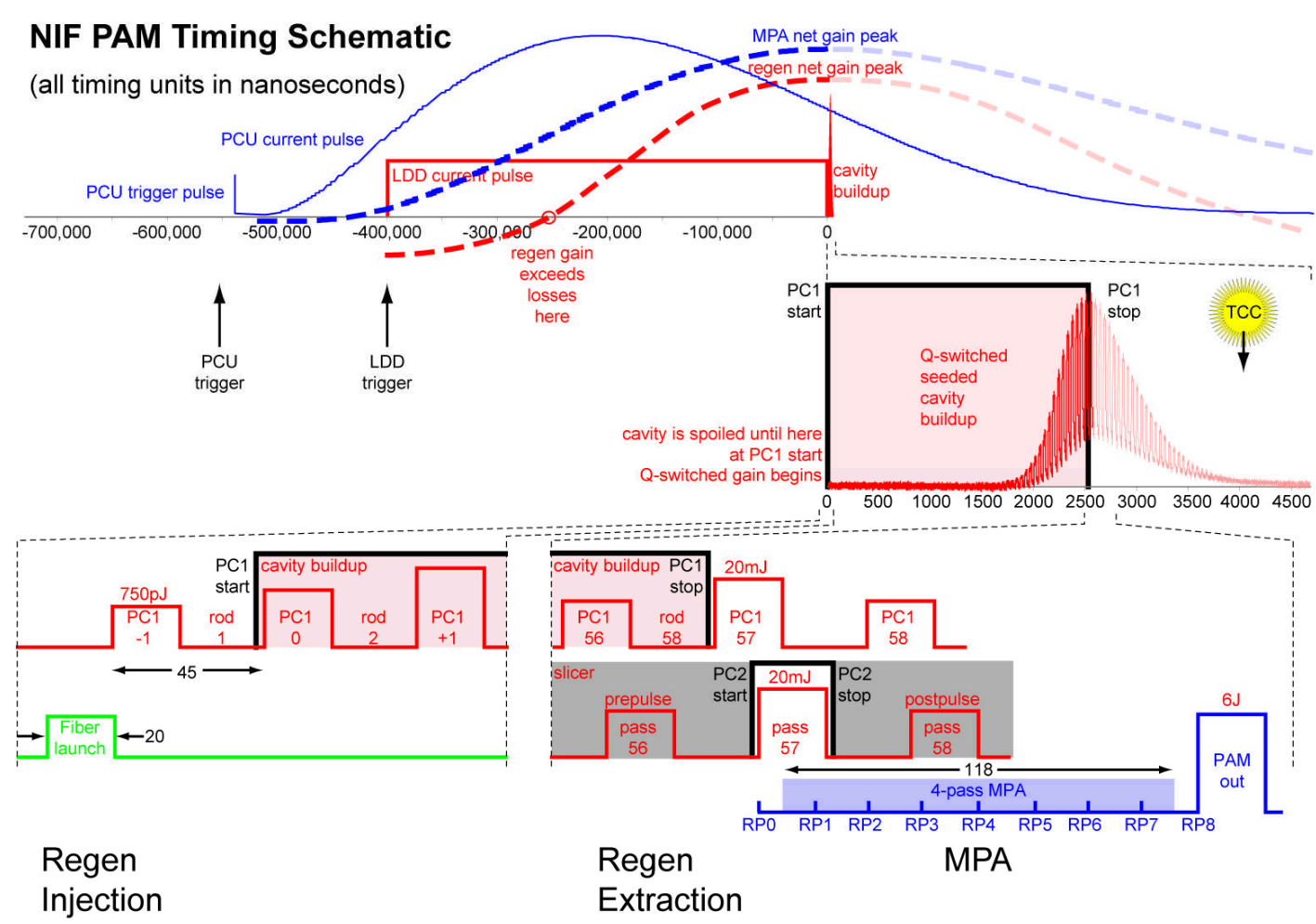

Overview of PAM order of events involved in firing a shot. All quantities are in nanoseconds. Note the existence of 3 separate timescales broken out covering nanoseconds, microseconds and milliseconds. 


\section{PAM and Beamline Splits}

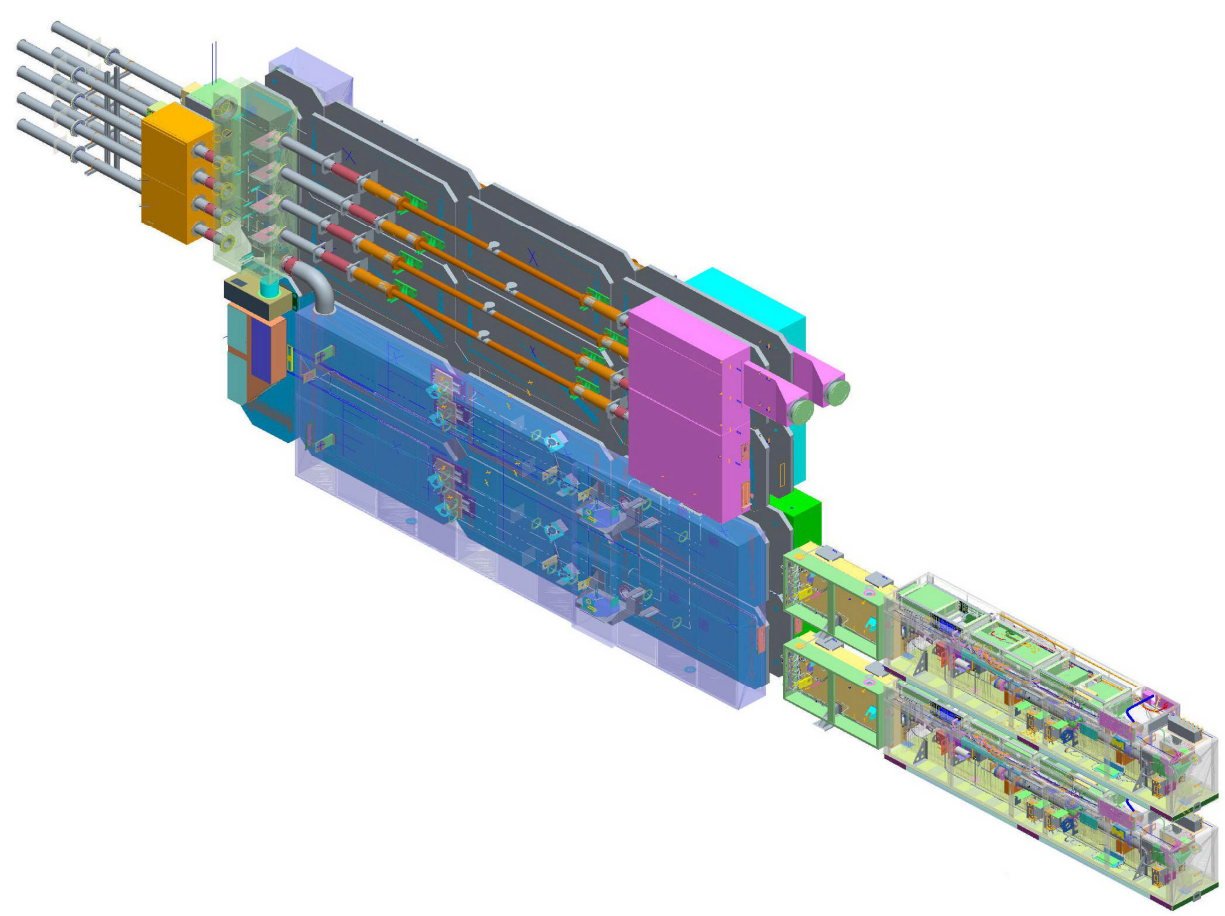

CAD rendering of a bundle of 2 PAMs (lower right) in relation to the branching optics feeding 8 main beamlines downstream (upper left). This is one of 24 modular subunits illustrated in whole in figure 1 . 


\section{PAM Installation Photographs}

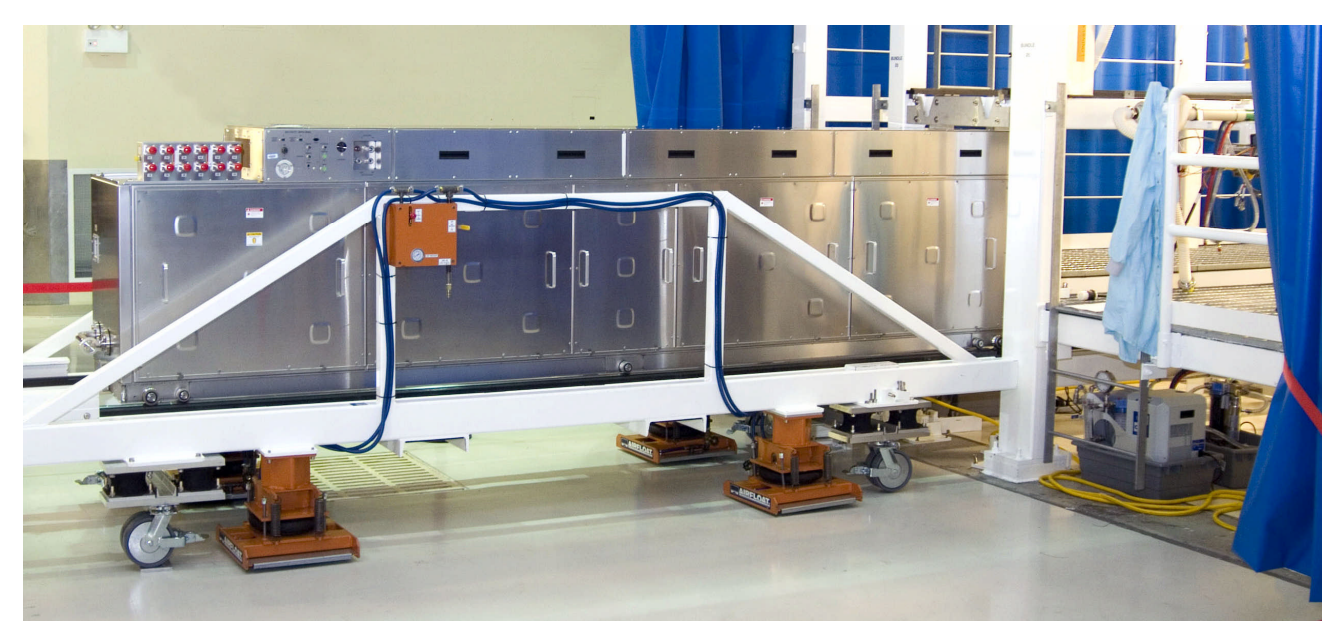

A PAM being installed into NIF laser bay. 


\section{PAM Regen Fluence Profiles}
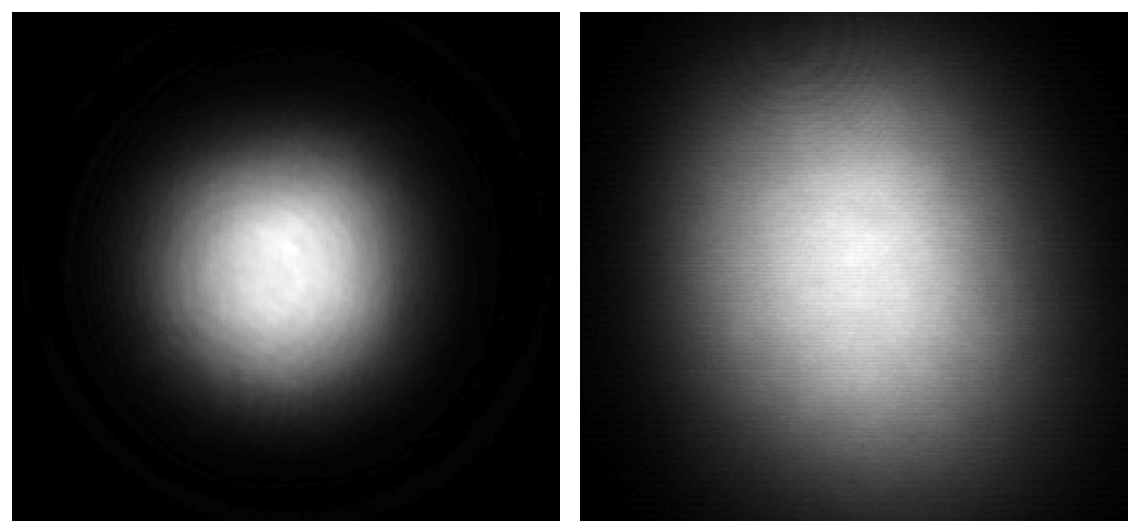

Regenerative Amplifier mode profile images in a) the rod and at b) the output prior to the $20 \mathrm{X}$ beam expander that back illuminates the beam shaping masks. These clean images illustrate the uniformity of the beam 
Appendix C

Multimedia 